\title{
Number of Foci Increases Risk of Central Compartment Lymph Node Metastasis in Papillary Thyroid Cancer
}

\author{
Dapeng $\mathrm{Li}^{1 \#}$, Mengran Tian ${ }^{1 \#}$, Xiukun Hou${ }^{1}$, Linfei Hu${ }^{1}$, Yanhui Zhang ${ }^{2}$, Xiangqian Zheng ${ }^{1}$, Ming \\ $\mathrm{Gao}^{3 *}$ \\ ${ }^{1}$ Department of Thyroid and Neck Oncology, Tianjin Medical University Cancer Institute and Hospital, National Clinical \\ Research Center for Cancer; Key Laboratory of Cancer Prevention and Therapy, Tianjin's Clinical Research Center for Cancer, \\ Tianjin, China
}

${ }^{2}$ Department of Pathology, Tianjin Medical University Cancer Institute and Hospital, National Clinical Research Center for Cancer, Key Laboratory of Cancer Prevention and Therapy, Tianjin's Clinical Research Center for Cancer, Tianjin, China

${ }^{3}$ Tianjin Union Medical Center, Tianjin, China

\#These authors contributed equally to this work

*Corresponding author: Ming Gao, Tianjin Union Medical Center, No.190 Jieyuan Road, Hongqiao District, Tianjin, 300121, China

\section{ARTICLE INFO}

Received: 㹃 September 03, 2021

Published: 幽 September 14, 2021

Citation: Dapeng Li, Mengran Tian, Xiukun Hou, Linfei Hu, Ming Gao, et al., Number of Foci Increases Risk of Central Compartment Lymph Node Metastasis in Papillary Thyroid Cancer. Biomed J Sci \& Tech Res 38(4)-2021. BJSTR. MS.ID.006198.

Keywords: Papillary Thyroid Microcarcinoma; Multifocality; Lymph Node Metastasis

\section{ABSTRACT}

Objectives: Multifocality is one of the prominent characteristics of papillary thyroid cancer (PTC), however the management strategy for multifocal PTC remains controversial. Moreover, evaluation of impact of multifocality in a quantitative way rather than a qualitative way still needs to be elucidated.

Patients and Methods: Medical records of 1034 patients with PTC who underwent total or hemi-thyroidectomy were reviewed at the authors' institution between April 2013 to September 2013. The laterality, number and size of each tumor foci, number of levels VI lymph nodes metastasis (LNM) and other potential risk factors were recorded.

Results: Male gender, tumor size larger than $1 \mathrm{~cm}$, multifocality, diffuse calcification were risk factors for level VI LNM by univariant and multivariable analysis. Moreover, level VI LNM rate is increased proportionally with the increasing number of foci. The LNM rate is $102 / 474$ (21.5\%), 46/135 (34.1\%), 14/35 (40\%), and $4 / 7$ (57.1\%) for unilateral PTMC patients with 1 to 4 foci, and 15/30 (50\%), 15/24 (62.5\%), 7/10 (70\%), 5/6 (83.3\%), 2/2 (100\%) for bilateral PTC patients with 2 to 6 foci. Interestingly, we found that the LNM rate for multifocal PTMCs $(6 \mathrm{~mm} \leq l$ argest diameter $\leq 10 \mathrm{~mm})$ is comparable to unilateral unifocal PTCs (largest diameter $>1 \mathrm{~cm}, 52 / 122,42.6 \%$, vs. 21/58, 36.2\%; $\mathrm{P}=0.413$ ).

Conclusion: Our study suggests that multifocality as a sign of a higher propensity for lymph node metastasis. Multifocal PTMC with $6 \mathrm{~mm} \leq$ largest diameter $\leq 10 \mathrm{~mm}$ should be treated with caution considering the possible high lymph node metastasis rate.

\section{Introduction}

The incidence of papillary thyroid carcinoma (PTC) has increased in a dramatic pattern in recent years [1,2]. PTC often presents with multifocal tumors, i.e., presence of two or more non-contiguous tumor foci either unilaterally or bilaterally, which is one of the most prominent characteristics of PTC. It is well established that multifocality represents a higher propensity for a more advanced staging, and multifocality per se is a qualitative parameter to assess the risk for LNM. However, it is not easy to quantify its effects on pathological outcome and clinical features. 
The prevalence of the multifocal PTC ranged from 32\% to $39 \%$ in large series of PTC [3-5]. In clinics, multifocality is often empirically treated as a high-risk factor for aggressiveness of PTC and reported to be associated with extensive LNM [4], higher recurrence rate [5], and more aggressive biological behavior [6,7], compared with unifocal PTC. However, inconsistent and even contradictory findings also exist $[4,8,9]$. It is well accepted that active surveillance should be recommended to patient with microcarcinoma at low risk. However, a clinical dilemma is how do physicians handle with multifocal microcarcinoma which all the foci are less than $1 \mathrm{~cm}$ in diameter. In other words, whether multifocality could be used as a quantified parameter rather than a qualitative parameter need to be addressed. Moreover, although prophylactic central compartment neck dissection is not routinely recommended according to ATA guidelines, insidious LNM do exist and be the cause for the recurrence of PTC. Thus, is it safe to recommend not receiving central compartment neck dissection for all patients with multifocal papillary thyroid microcarcinoma?

In this study, we retrospectively analyzed 1034 patients with papillary thyroid carcinoma to evaluate the how the multifocality affects the central compartment LNM and try to quantify the multifocality to further predict the possible insidious central compartment LNM.

\section{Material and Methods}

\section{Patients}

Patients diagnosed with papillary thyroid cancer (classic type) between April 2013 to September 2013 in Tianjin Medical University Cancer Institute and Hospital were recruited. Medical records, ultrasonographic and histopathological reports were reviewed. Patients with histological variant (follicular, tall cell, trabecular or others) or patients whose tumor is in the isthmus were not included. Sex, age, tumor size, bilateral disease, tumor foci number, and cervical LNM were determined in each patient. Ethics approval was obtained from The Institutional Review Board of Tianjin Medical University Cancer Institute and Hospital (approval: BC2021023), and the requirements for patient approval and informed consent were waived because of the retrospective nature of the study.

\section{Data Collection/ Definition of Parameter}

Bilateral disease was defined as the presence of PTC foci in the right and left lobes of the thyroid gland. The size of the largest focus of PTC was used for analysis. Multifocal disease was defined as the presence of two or more foci of PTC.

\section{Determination of Number of Tumor Foci and Tumor Size}

To define the number tumor foci and tumor diameter of each tumor, ultrasonographic and histopathological reports were reviewed by two independent researchers. The number of tumor foci is determined according to the histopathological reports. As for each tumor's largest diameter, we refer to the ultrasonographic reports for each tumor and record its ultrasonographic diameter. The largest diameter is recorded if three-dimensional data is given by the ultrasonographers.

\section{Statistical Analysis}

Statistical analysis was performed using IBM SPSS Statistics for Windows v21 (IBM Corp., Armonk, NY). Univariate analysis was performed using Chi-squared test or Fisher's exact tests for categorical variables, Student's t-tests for normally distributed data, and Mann-Whitney U-tests for continuous variables that were not normally distributed. Cox regression analyses was used for univariate and multivariate analysis. All p-values represented were two-sided and considered statistically significant when $\mathrm{p}<0.05$.

\section{Results}

\section{Clinical and Pathological Features}

Table 1: Clinical characteristics of study patients.

\begin{tabular}{|c|c|}
\hline Variable & Value (\%) \\
\hline Age & $46 \pm 9.4$ \\
\hline \multicolumn{2}{|l|}{ Gender } \\
\hline Male & $229(22.1 \%)$ \\
\hline Female & $805(77.9 \%)$ \\
\hline \multicolumn{2}{|l|}{ Size of largest focus } \\
\hline Average & 7.75 \\
\hline Median & 7 \\
\hline \multicolumn{2}{|l|}{ Surgical extent of primary tumor } \\
\hline Unilateral / Lobectomy & $725(70.1 \%)$ \\
\hline Bilateral/ Total thyroidectomy & $309(29.9 \%)$ \\
\hline \multicolumn{2}{|l|}{ Number of foci } \\
\hline 1 & $532(51.5 \%)$ \\
\hline 2 & $291(28.1 \%)$ \\
\hline 3 & $139(13.4 \%)$ \\
\hline 4 & $47(4.5 \%)$ \\
\hline$\geq 5$ & $25(2.4 \%)$ \\
\hline \multicolumn{2}{|l|}{ Level VI LNM (+) } \\
\hline Unilateral disease & $192(26.5 \%)$ \\
\hline Bilateral disease & $141(45.6 \%)$ \\
\hline Lateral neck LNM (+) & $48(4.6 \%)$ \\
\hline \multicolumn{2}{|l|}{ LN harvested } \\
\hline Average & 3.42 \\
\hline Median & 3 \\
\hline
\end{tabular}

Abbreviations: LNM, lymph node metastasis

A total of 1034 patients were diagnosed as PTC or PTMC in our department during April 2013 to September 2013. The mean 
age was 46 years (range, 18-76), of which 229 (22.1\%) were male and 805 were female (77.9\%). The median tumor size was $7 \mathrm{~mm}$ (range, 1-45). Multifocal cancer lesions were found in 502 patients (48.5\%), in which the number of patients with 2 to 4 foci were $291(28.1 \%), 139(13.4 \%)$, and $47(4.5 \%)$, respectively, with $25(2.4 \%)$ patients with $\geq 5$ foci. Of all the multifocal PTMCs, 273 (57.2\%) were bilateral and 177 (42.8\%) were unilateral. The level VI LNM rate for unilateral and bilateral cancer patients were $26.5 \%$ and $45.6 \%$, respectively. The clinical and pathological features of these patients were summarized in Table 1.

\section{Factors Associated with Central Compartment LNM}

Univariate analysis was done to assess the risk factors of central compartment LNM. As for all the patients included, male gender, tumor size larger than $1 \mathrm{~cm}$, multifocality, bilateral disease, diffuse calcification were risk factors for central compartment LNM Table 2. Interestingly, when multifocal diseases were considered (including both multifocal PTCs and multifocal PTMCs), bilateral disease was no longer a risk factor $(\mathrm{P}=0.053, \mathrm{P}=0.095$, respectively). We then calculated the independent risk factor for LNM by multivariate analysis, we found that male gender, multifocality, diameter larger than $1 \mathrm{~cm}$, diffuse calcification on ultrasonography are all independent risk factors for LNM. However, age and bilaterality are not independent risk factors for LNM Table 3.

Central compartment LNM rate is associated with multifocality irrespective of unilateral or bilateral disease.

Table 2: Factors predictive of lymph node metastasis in the univariable analysis.

\begin{tabular}{|c|c|c|c|c|}
\hline \multicolumn{5}{|c|}{ Lymph Node Metastasis } \\
\hline Factors & All Patients & LNM- & LNM+ & $P$ value \\
\hline \multicolumn{5}{|l|}{ Gender } \\
\hline Female & 805 & $558(69.3 \%)$ & $247(30.7 \%)$ & \multirow{2}{*}{0.000} \\
\hline Male & 229 & $128(55.9 \%)$ & $101(44.1 \%)$ & \\
\hline \multicolumn{5}{|l|}{ Age (year) } \\
\hline$<55$ & 842 & $551(65.4 \%)$ & $291(34.6 \%)$ & \multirow{2}{*}{0.197} \\
\hline$\geq 55$ & 192 & $135(70.3 \%)$ & $57(29.7 \%)$ & \\
\hline \multicolumn{5}{|l|}{ Tumor size } \\
\hline Microcarcinoma & 888 & $617(69.5 \%)$ & $271(30.5 \%)$ & \multirow{2}{*}{0.000} \\
\hline Non-microcarcinoma & 146 & $69(47.3 \%)$ & $77(52.7 \%)$ & \\
\hline \multicolumn{5}{|l|}{ Focality } \\
\hline Unifocal & 532 & $409(76.9 \%)$ & $123(23.1 \%)$ & \multirow{2}{*}{0.000} \\
\hline Multifocal & 502 & $277(55.2 \%)$ & $225(44.8 \%)$ & \\
\hline \multicolumn{5}{|l|}{ Bilateral disease } \\
\hline No & 725 & $526(72.6 \%)$ & $199(27.4 \%)$ & \multirow{2}{*}{0.000} \\
\hline Yes & 309 & $160(51.8 \%)$ & $149(48.2 \%)$ & \\
\hline \multicolumn{5}{|c|}{ Multifocal PTCs and PTMCs } \\
\hline Unilateral disease & 193 & $117(60.6 \%)$ & $76(39.4 \%)$ & \multirow{2}{*}{0.053} \\
\hline Bilateral disease & 309 & $160(51.8 \%)$ & $149(48.2 \%)$ & \\
\hline \multicolumn{5}{|l|}{ Multifocal PTMCs } \\
\hline Unilateral disease & 177 & $113(63.8 \%)$ & $64(36.2 \%)$ & \multirow{2}{*}{0.095} \\
\hline Bilateral disease & 237 & $132(55.7 \%)$ & $105(44.3 \%)$ & \\
\hline \multicolumn{5}{|l|}{ Diffuse calcification } \\
\hline Yes & 3 & $0(0 \%)$ & $3(100 \%)$ & \multirow{2}{*}{0.038} \\
\hline No & 1031 & $686(66.5 \%)$ & $345(33.5 \%)$ & \\
\hline
\end{tabular}

Abbreviations: PTMC, papillary thyroid microcarcinoma; PTC, papillary thyroid carcinoma; LNM, lymph node metastasis. 
Table 3: Factors predictive of lymph node metastasis in the multi-variable analysis.

\begin{tabular}{|c|c|c|c|c|c|c|}
\hline Factors & B & SE & Wald & HR & P & 95\% CI \\
\hline Gender (male/female) & 0.620 & 0.160 & 15.041 & 1.859 & $<0.001$ & $1.359-2.543$ \\
\hline Multifocality (yes/no) & 0.801 & 0.184 & 19.047 & 2.228 & $<0.001$ & $1.555-3.192$ \\
\hline Diameter $(>1 \mathrm{~cm}$ vs $\leq 1 \mathrm{~cm})$ & 0.812 & 0.190 & 18.323 & 2.252 & $<0.001$ & $1.553-3.266$ \\
\hline Age $(\geq 55$ vs $<55$ years) & -0.214 & 0.182 & 1.377 & 0.808 & 0.214 & $0.565-1.154$ \\
\hline Bilaterality (yes/no) & 0.250 & 0.192 & 1.695 & 1.284 & 0.193 & $0.881-1.871$ \\
\hline
\end{tabular}

In PTCs that larger than $1 \mathrm{~cm}$ in diameter, the LNM rate of unifocal PTC is $36.2 \%$ (21/58), whereas the LNM rate of multifocal PTC is much higher at $63.6 \%(56 / 88, \mathrm{P}=0.001)$. We then analyzed the effect of bilaterality on central compartment LNM. Of note, the LNM rate of unilateral multifocal PTC is $75 \%(12 / 16)$, which is comparable to the LNM of bilateral multifocal PTC $61.1 \%(44 / 72$, $\mathrm{P}=0.296)$. Similar results were observed in PTMC patients. The LNM rate of unifocal PTMC is $21.5 \%(102 / 474)$, whereas the LNM of multifocal PTMC is $40.8 \%$ (169/414), in which LNM of unilateral multifocal PTMC is $36.2 \%$ (64/177), which is comparable to the LNM rate of bilateral multifocal PTMC 44.3\% (105/237, P=0.095), as summarized in Table 2.

\section{Number of Foci Increases the LNM Rate}

The above results showed that multifocality is a risk factor for increased LNM rate irrespective of unilateral or bilateral disease. This finding suggests that multifocality might be interpreted in the setting of one "thyroid lobe", rather than "one thyroid gland". Thus, the effect of the number of PTC foci on central compartment LNM was further examined. As shown in Table 4, for all the unilateral PTMC, 102 of 474 (21.5\%) patients with unifocal PTMC had level VI lymph node metastases. Whereas 46/135 (34.1\%) patients with two foci of PTMC were found to have positive lymph nodes. The proportion of patients with positive level VI nodes increased in the group with three foci, where $14 / 35$ (40\%) patients were found to be positive. In patients with 4 foci, $4 / 7$ (57.1\%) were found to have positive lymph nodes in level VI. Interestingly, a gradual increase in LNM proportion is observed, with respect to foci number, as displayed in Figure 1. In bilateral PTMC patients, 41 of 112 (36.6\%) patients with two foci bilateral PTMC had level VI lymph node metastases. Whereas patients with 3 to 7 foci of bilateral PTMC were found to have positive LNM were $41 / 78$ (52.6\%), 13/30 (43.3\%), 6/11 (54.5\%), 2/3(66.7\%), 2/3(66.7\%), respectively.

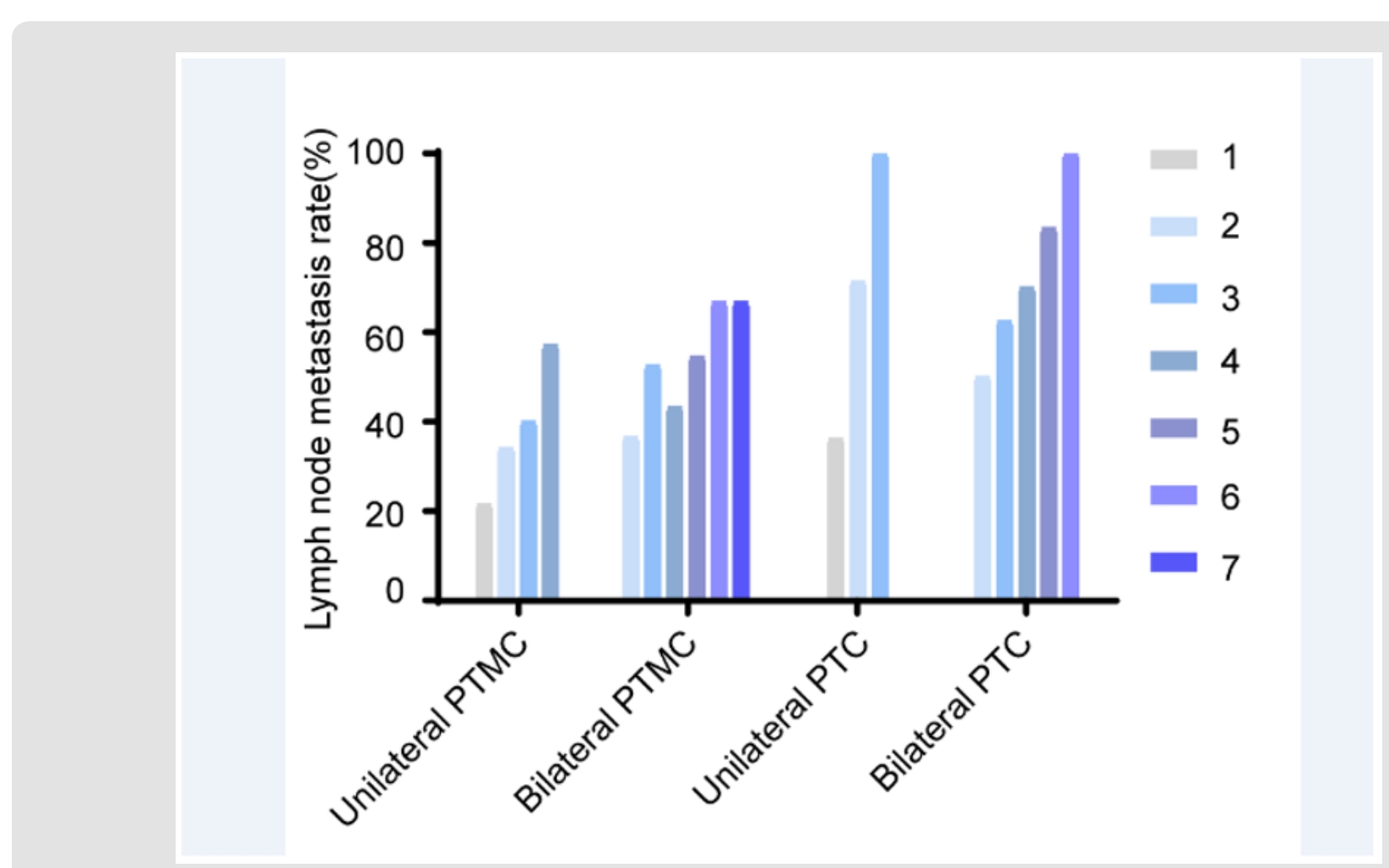

Figure 1: Central lymph node metastasis rate is increased with respect to increased foci number. For all unilateral or bilateral PTMCs and PTCs, the LNM rate is increased in accord with the increased foci number. 
Table 4: Lymph node metastasis rate at various number of foci.

\begin{tabular}{|c|c|c|c|c|c|c|c|}
\hline & \multicolumn{7}{|c|}{ Number of foci } \\
\hline & 1 & 2 & 3 & 4 & 5 & 6 & 7 \\
\hline Unilateral PTMC & $21.5 \%$ & $34.1 \%$ & $40 \%$ & $57.1 \%$ & - & - & - \\
\hline Bilateral PTMC & - & $36.6 \%$ & $52.6 \%$ & $43.3 \%$ & $54.5 \%$ & $66.7 \%$ & $66.7 \%$ \\
\hline Unilateral PTC & $36.2 \%$ & $71.4 \%$ & $100 \%$ & - & - & - & - \\
\hline Bilateral PTC & - & $50 \%$ & $62.5 \%$ & $70 \%$ & $83.3 \%$ & $100 \%$ & - \\
\hline
\end{tabular}

Abbreviations: PTMC, papillary thyroid microcarcinoma; PTC, papillary thyroid carcinoma

A similar result was also observed in PTC patients with largest diameter larger than $10 \mathrm{~mm}$. As for unilateral PTC patients with largest diameter larger than $10 \mathrm{~mm}$, the LNM proportion is $21 / 58$ (36.2\%), 10/14 (71.4\%) and 2/2 (100\%) for unilateral PTC patients with 1 to 3 foci. For bilateral PTC patients with largest diameter larger than $10 \mathrm{~mm}$, the LNM proportion is $15 / 30(50 \%)$, 15/24 (62.5\%), 7/10 (70\%), 5/6 (83.3\%), 2/2 (100\%) for bilateral PTC patients with 2 to 6 foci Table 4 .

\section{LNM Rate for Multifocal PTMC Is Comparable to Unifocal PTC When Ptmcs Largest Diameter is Between 6-10 mm}

To further assess the effect of multifocality on LNM, patients were stratified according to the largest diameter. Patients were divided into three groups: A. largest diameter is $\leq 5 \mathrm{~mm}$; B. $6 \mathrm{~mm} \leq$ largest diameter $\leq 10 \mathrm{~mm}$; C. largest diameter $>10 \mathrm{~mm}$. As shown in Table 5, in group A, the LNM proportion in unilateral unifocal PTMC, unilateral multifocal PTMC and bilateral multifocal is $27 / 184$ (14.7\%), 12/55 (21.8\%) and 27/103 (26.2\%). In group B, the LNM proportion in unilateral unifocal PTMC, unilateral multifocal PTMC and bilateral multifocal is 75/290 (25.9\%), 52/122 (42.6\%) and $142 / 311$ (45.7\%). In group C, the LNM proportion in unilateral unifocal PTC, unilateral multifocal PTC and bilateral multifocal PTC is $21 / 58$ (36.2\%), $12 / 16(75.0 \%)$ and $44 / 72$ (61.1\%). For unilateral PTMCs and PTCs, the LNM proportion in patients from group A, B and $\mathrm{C}$ were 12/55 (21.8\%), 52/122 (42.6\%) and 12/16 (75\%). For both unilateral and bilateral PTMCs and PTCs, the LNM proportion in patients from group A, B and C were 27/103 (26.2\%), 142/311 (45.7\%) and 56/88 (63.6\%). Interestingly, when we compare the risk of LNM for multifocal PTMCs, we found that the LNM rate for multifocal PTMCs in group B $(6 \mathrm{~mm} \leq$ largest diameter $\leq 10 \mathrm{~mm})$ is comparable to unilateral unifocal PTCs whose largest diameter is greater than $1 \mathrm{~cm}(52 / 122,42.6 \%$, vs. $21 / 58,36.2 \%$; $\mathrm{P}=0.413)$, indicating that multifocal PTMCs might have the same propensity to LNM with unifocal PTC which largest diameter is greater than $1 \mathrm{~cm}$

Table 5: Lymph node metastasis rate according to largest tumor diameter.

\begin{tabular}{|c|c|c|c|}
\hline & Group A & Group B & Group C \\
\hline Unilateral unifocal TC & $27 / 184(14.7 \%)$ & $75 / 290(25.9 \%)$ & $21 / 58(36.2 \%)$ \\
\hline Unilateral multifocal TC & $12 / 55(21.8 \%)$ & $52 / 122(42.6 \%)$ & $12 / 16(75.0 \%)$ \\
\hline Bilateral multifocal TC & $27 / 103(26.2 \%)$ & $142 / 311(45.7 \%)$ & $44 / 72(61.1 \%)$ \\
\hline
\end{tabular}

Group A: PTMC's largest diameter is $\leq 5 \mathrm{~mm}$; Group B: $6 \mathrm{~mm} \leq$ largest diameter $\leq 10 \mathrm{~mm}$; Group C: largest diameter $>10 \mathrm{~mm}$.

Abbreviations: TC: thyroid cancer

\section{Discussion}

Previously, multifocality has been implicated as a qualitative parameter to assess the risk for central compartment LNM. However, few studies have shown how the number of foci affect the LNM. In this study, the risk factors for lymph node metastasis were studied, then the effect of multifocality was further investigated between the number of PTC foci and LNM to central compartment. Multifocality is a common clinical characteristic of PTC and has been reported in $18-87 \%$ of patients with PTC [10] and was seen in $48.5 \%$ of patients in the present study. Our results show that the risk of level VI lymph metastasis increases proportionally with the number of tumor foci, both in PTMCs and PTCs which diameter is larger than $1 \mathrm{~cm}$. When the number of foci reaches 3 or more, the level VI LNM rate exceeds $50 \%$ or higher $(52.6 \%$ and $62.5 \%$ for bilateral PTMC and bilateral PTC). And even 100\% in the presence of 6 foci. Ayham et al reported a similar result in the presence of 3 or more foci, except that no appreciable difference in level VI lymph node metastasis in patients with one and two foci [11]. These findings identify multifocality in PTC as an indicator of tumor aggressiveness, manifesting in a higher propensity for locoregional metastasis.

Both univariate and multivariate analysis identified male gender, tumor size larger than $1 \mathrm{~cm}$, multifocality, diffuse calcification as significant predictors of level VI lymph node 
metastasis in the study population. These findings are consistent with previously published studies identifying risk factors for level VI metastasis in PTC [12-14]. Of note, our data indicated a rare phenomenon diffuse calcification as significant predictors of level VI lymph node metastasis. Diffuse calcification has been implicated with thyroid malignancy $[15,16]$. In our study, the LNM rate for the PTC with diffuse calcification is $3 / 3(100 \%)$, although the case number is small, it still strongly indicates that PTC patients with diffuse calcification under ultrasonography has a higher propensity for locoregional metastasis. Another interesting finding is that, when we compare the LNM rate between multiple PTMCs $(6 \mathrm{~mm} \leq$ largest diameter $\leq 10 \mathrm{~mm})$ and unifocal PTC which diameter is larger than $1 \mathrm{~cm}$, there is no significant difference between these two groups. This finding indicates that multifocal PTMCs with $6 \mathrm{~mm} \leq$ largest diameter $\leq 10 \mathrm{~mm}$ has the same propensity for LNM in central compartment, compared with unifocal PTC with largest diameter $\geq 10 \mathrm{~mm}$. Other studies have shown concordant result that multifocal PTMC with relative bigger foci is at higher risk of LNM although it might not be clinically evident. Zhao et al. has proposed the concept of aggregate diameter (AD) to better assess the tumor burden $[17,18]$. Abbas et al. also pointed out that the sum up of the diameter of each tumor foci $>10 \mathrm{~mm}$ confers a similar risk of aggressive histopathological behavior with unifocal PTC greater than $10 \mathrm{~mm}$ [19].

In conclusion, this study provides evidence that multifocal PTCs are at higher risk of LNM, which tendency might increase in the presence of over 4 foci when compared with unifocal and bifocal disease. In addition, multifocal PTMCs with largest foci between $6 \mathrm{~mm}$ to $10 \mathrm{~mm}$ might have the same tendency to PTC which diameter is $>10 \mathrm{~mm}$. Thus, multifocal thyroid PTMCs should not be managed as conventional PTMCs at low risk, thereby prompting more aggressive treatments.

\section{Acknowledgments}

This work was partially supported by grants from National Natural Science Foundation of China (Grant Nos. 81872169), Tianjin key research and development program science and technology support key projects (Grant No.17YFZCSY00690), Tianjin Municipal Science and technology project (Grant No. 19JCYBJC27400) and Beijing-Tianjin-Hebei Basic Research Cooperation Project (Grant No. 20JCZXJC00120).

\section{Disclosure}

The author reports no conflicts of interest in this work.

\section{References}

1. Haugen BR, Alexander EK, Bible KC, Gerard M Doherty 4, Susan J Mandel, et al. (2016) 2015 American Thyroid Association Management
Guidelines for Adult Patients with Thyroid Nodules and Differentiated Thyroid Cancer: The American Thyroid Association Guidelines Task Force on Thyroid Nodules and Differentiated Thyroid Cancer. Thyroid: official journal of the American Thyroid Association 26(1): 1-133.

2. Gao M, Ge M, Ji Q Ruochuan Cheng, Hankui Lu, et al. (2016) Chinese expert consensus and guidelines for the diagnosis and treatment of papillary thyroid microcarcinoma. Cancer Biol Med 14(3): 203-211.

3. Shi X, Liu R, Basolo F, Riccardo Giannini, Xiaopei Shen, et al. (2016) Differential Clinicopathological Risk and Prognosis of Major Papillary Thyroid Cancer Variants. The Journal of clinical endocrinology and metabolism 101(1): 264-274.

4. Kim HJ, Sohn SY, Jang HW, Kim SW, Chung JH, et al. (2013) Multifocality, but not bilaterality, is a predictor of disease recurrence/persistence of papillary thyroid carcinoma. World J Surg 37(2): 376-384.

5. Kim KJ, Kim SM, Lee YS, Chung WY, Chang HS, et al. (2015) Prognostic significance of tumor multifocality in papillary thyroid carcinoma and its relationship with primary tumor size: a retrospective study of 2,309 consecutive patients. Annals of surgical oncology 22(1): 125-131.

6. Zhang W, Jiao D, Liu B, Sun S (2016) Analysis of Risk Factors Contributing to Recurrence of Papillary Thyroid Carcinoma in Chinese Patients Who Underwent Total Thyroidectomy. Medical science monitor: international medical journal of experimental and clinical research 22: 1274-1279.

7. Grogan RH, Kaplan SP, Cao H, Roy E Weiss, Leslie J Degroot, et al. (2013) A study of recurrence and death from papillary thyroid cancer with 27 years of median follow-up. Surgery 154(6): 1436-1446; discussion 1446-1437.

8. Leboulleux S, Rubino C, Baudin E, Bernard Caillou, Dana M Hartl, et al. (2005) Prognostic factors for persistent or recurrent disease of papillary thyroid carcinoma with neck lymph node metastases and/or tumor extension beyond the thyroid capsule at initial diagnosis. The Journal of clinical endocrinology and metabolism 90(10): 5723-5729.

9. Neuhold N, Schultheis A, Hermann M, Krotla G, Koperek O, et al. (2011) Incidental papillary microcarcinoma of the thyroid--further evidence of a very low malignant potential: a retrospective clinicopathological study with up to 30 years of follow-up. Annals of surgical oncology 18(12): 3430-3436.

10. Katoh R, Sasaki J, Kurihara H, Suzuki K, Iida Y, et al. (1992) Multiple thyroid involvement (intraglandular metastasis) in papillary thyroid carcinoma. A clinicopathologic study of 105 consecutive patients. Cancer $70(6)$ : 1585-1590.

11. Al Afif A, Williams BA, Rigby MH, Martin J Bullock, S Mark Taylor, et al. (2015) Multifocal Papillary Thyroid Cancer Increases the Risk of Central Lymph Node Metastasis. Thyroid: official journal of the American Thyroid Association 25(9): 1008-1012.

12. Bian X, Chen H, Ye X, Tang PJ (2010) Role of level VI lymph nodes metastasis in cervical metastasis of patients with papillary thyroid cancer. Zhonghua Er Bi Yan Hou Tou Jing Wai Ke Za Zhi 45(8): 664-668.

13. Yang Y, Chen C, Chen Z, Jiachun Jiang, Yizuo Chen, et al. (2014) Prediction of central compartment lymph node metastasis in papillary thyroid microcarcinoma. Clin Endocrinol 81(2): 282-288.

14. Kim K, Kim E, Yoon J, Han K, Moon H, et al. (2013) Preoperative prediction of central lymph node metastasis in thyroid papillary microcarcinoma using clinicopathologic and sonographic features. World J Surg 37(2): 385-391.

15. Ning CP, Ji QL, Fang SB, Wang HQ, Zhong YM, et al. (2018) Distribution patterns of microcalcifications in suspected thyroid carcinoma: a classification method helpful for diagnosis. European radiology 28(6): 2612-2619. 
16. Ha EJ, Baek JH, Lee JH (2017) Diffuse Microcalcifications of Only the Thyroid Gland Seen on Ultrasound: Clinical Implication and Diagnostic Approach. Annals of surgical oncology 24(Suppl 3): 641.

17. Wang P, Wang Y, Miao C, Xing Yu, Haichao Yan, et al. (2017) Defining a New Tumor Dimension in Staging of Papillary Thyroid Carcinoma. Annals of surgical oncology 24(6): 1551-1556.

ISSN: 2574-1241

DOI: 10.26717/BJSTR.2021.38.006198

Ming Gao. Biomed J Sci \& Tech Res

(c) (P) This work is licensed under Creative

Submission Link: https://biomedres.us/submit-manuscript.php
18. Zhao Q Ming J, Liu C, Lan Shi, Xia Xu, et al. (2013) Multifocality and total tumor diameter predict central neck lymph node metastases in papillary thyroid microcarcinoma. Annals of surgical oncology 20(3): 746-752.

19. Tam AA, Özdemir D, Çuhacı N, Hüsniye Başer, Cevdet Aydın, et al. (2016) Association of multifocality, tumor number, and total tumor diameter with clinicopathological features in papillary thyroid cancer. Endocrine 53(3): 774-783.

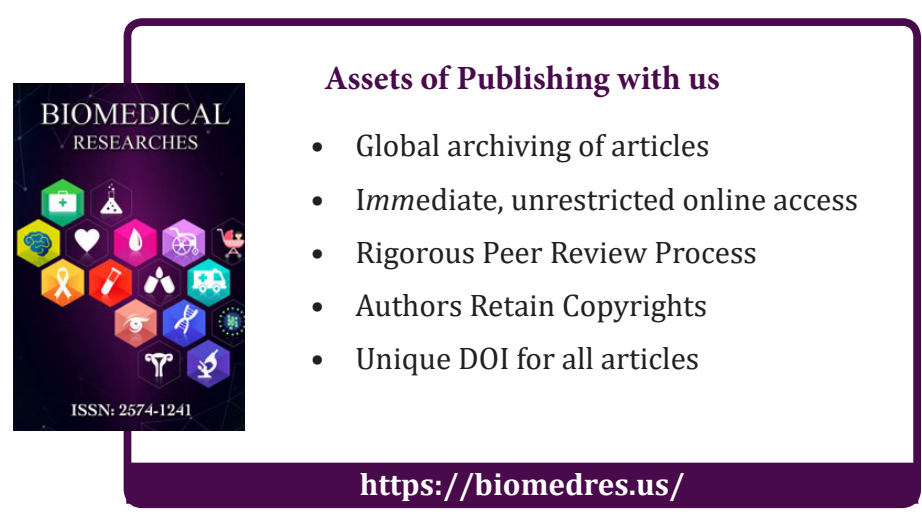

